\title{
Simplifying aortic arch surgery: open zone 2 arch with single branched thoracic endovascular aortic repair completion
}

\author{
Nimesh D. Desai, Ashley Hoedt, Grace Wang, Wilson Y. Szeto, Prasthanth Vallabhajosyula, Mary \\ Reinke, Joseph E. Bavaria \\ Division of Cardiovascular Surgery, University of Pennsylvania, Philadelphia, PA, USA \\ Correspondence to: Nimesh D. Desai, MD, PhD. Hospital of the University of Pennsylvania, Division of Cardiovascular Surgery, 3400 Spruce Street, \\ 6th Floor Silverstein, Philadelphia, PA 19104, USA. Email: nimesh.desai@uphs.upenn.edu.
}

\begin{abstract}
Background: Distal aortic complications from acute DeBakey I dissection repair are an important source of late morbidity and mortality. We present an early experience of using a novel single-branched thoracic aortic endograft in conjunction with open techniques to treat acute DeBakey I aortic dissection.

Methods: The patients in this series include five hyperacute dissections managed with a combined zone 2 partial arch replacement and placement of a zone 2 single subclavian branch endograft.

Results: There were no perioperative mortalities, strokes, or spinal cord ischemia in any patients at either stage of the procedure. At follow-up imaging, no patients had anterograde flow into the false lumen. All patients experienced false lumen thrombosis in the stented portion of the aorta.

Conclusions: This combination of open repair techniques and the use of a novel branched endograft resulted in excellent early outcomes in this pioneer series. Further investigation of these techniques in a prospective fashion is warranted.
\end{abstract}

Keywords: Aortic dissection; thoracic endovascular aortic repair (TEVAR); hybrid surgery; aortic arch replacement

Submitted Apr 23, 2018. Accepted for publication Apr 25, 2018.

doi: $10.21037 /$ acs.2018.05.08

View this article at: http://dx.doi.org/10.21037/acs.2018.05.08

\section{Background}

Acute type A aortic dissection is a challenging and lifethreatening condition encountered by cardiovascular surgeons. The paradigm of prompt diagnosis, transfer to an experienced surgical team, and expeditious surgery is well established $(1,2)$. However, the degree of complexity of the distal aortic repair in DeBakey Type I dissection is often highly variable among surgeons (3-8). In this context, the recent development of branched endograft technology has enabled new therapeutic options that may be able to simplify and combine them with open techniques, allowing for a more complete thoracic aortic repair. The current report describes a series of novel procedures enabling more complete treatment of acute DeBakey I aortic dissections presenting emergently. All of the procedures involve the use of a new single-branched thoracic aortic stent graft placed into zone 2 with a single side branch into the left subclavian artery (LSCA). Furthermore, such procedures represent the 'first-in-man' experiences of this novel technique.

\section{Methods \\ Patients}

The patients in this series include five hyperacute dissections managed with a combined zone 2 partial arch replacement and placement of a zone 2 single subclavian branch endograft. All acute patients had large tears either in the aortic arch or proximal descending aorta, necessitating more complex arch repair.

\section{Operative techniques}

The zone 2 arch replacement procedure was performed in five 


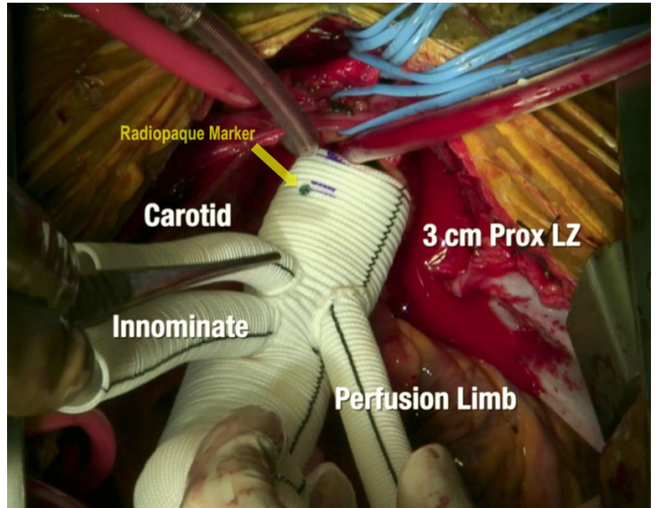

Figure 1 Two-branched arch graft with perfusion limb and radiopaque marker for future TEVAR landing. TEVAR, thoracic endovascular aortic repair.

patients in the hyper acute phase ( $<12$ hours of presentation) and two patients in chronic residual dissection following previous dissection repair. In two of these cases (one acute, one chronic) the ascending aorta was cannulated directly over the wire using transesophageal echocardiographic (TEE) guidance and a Seldinger technique. In the remaining cases, axillary artery cannulation was performed using a graft sewn to the axillary artery. Antegrade cerebral perfusion (ACP) was employed using the innominate artery with a balloontipped catheter in central cannulation cases, and via the axillary artery in the others. The left common carotid artery was clamped during the ACP to maintain pressurization of the cerebral circulation. The zone 2 arch was performed using a custom designed polyethylene terephthalate graft (Vascutek Gelweave) (Figure 1) with a 12-mm branch for the innominate artery and an $8-\mathrm{mm}$ graft for the left common carotid. A separate perfusion limb is present for graft recannulation. There are radiopaque markers on the graft to facilitate future thoracic endovascular aortic repair (TEVAR) docking. The arch was replaced with the initiation of unilateral ACP, clamping of the left carotid artery, and resection of the arch to zone 2. The layers of the aorta were reconstructed with felt neo-media reconstruction as we have previously described. The two-branched surgical graft was then sewn to the repaired zone 2 aorta using a 3-0 polypropylene suture. Following this, the left carotid artery was anastomosed, the graft was recannulated, and circulation was resumed to the body, left carotid, and LSCA. ACP was maintained to the innominate/axillary artery while it was reconstructed with the $12 \mathrm{~mm}$ limb.

The completion TEVAR was performed using the
GORE TBE single branched thoracic endograft device. This device is currently under an FDA Early Feasibility Study protocol under the Investigational Device Exemptions (IDE) regulations $(9,10)$. The cases in this series were outside the indications of this Early Feasibility Study and were used under 'Compassionate' or 'Emergency' use indications. All patients signed an individualized consent form approved by the local Institutional Review Board to receive the investigational device and reports were filed on a timely basis to the FDA. Patients were followed up with routine pre-discharge, 3 months, 6 months, and yearly imaging, which is our standard clinical follow-up.

The device consists of a main aortic body component, similar to the Gore CTAG device but with a separate side branch portal. The main aortic component has two configurations, a longer $(>2.5 \mathrm{~cm})$ covered proximal segment with a $12-\mathrm{mm}$ portal ideal for either ascending (zone 0 ) or graft landing zones, and a shorter $(<1.5 \mathrm{~cm})$ covered proximal segment with an $8-\mathrm{mm}$ portal ideal for native aorta zone 2 landings. Graft lengths are $10-15 \mathrm{~cm}$ depending on diameter. In this series, all cases were performed with the longer proximal covered segment with the $12 \mathrm{~mm}$ portal configuration. The side branches are tapered grafts up to 25 $\mathrm{mm}$ in diameter that are specifically designed to lock into the portal and maintain structural integrity in the setting of aortic motion from pulsatility and respiratory variation. There are also aortic cuffs of approximately $4 \mathrm{~cm}$ length for proximal extension if needed.

The portal was pre-cannulated prior to device insertion with a wire that was externalized through either the left brachial artery. The wire was externalized by snaring in the true lumen in the descending aorta. Intravascular ultrasound imaging (IVUS) was used to verify position of all wires in the true lumen. Care was taken to achieve appropriate rotational alignment of the portal along the greater curve of the aorta. Once the main aortic component is deployed, the branch was deployed over the externalized wire. The proximal seal zone, which were all in the surgical graft in this study, and portal were gently profile ballooned with compliant Gore Tri-lobe or Cook CODA balloons, respectively. Distal extension of the TEVAR with commercially available CTAG devices was then performed. Completed open repair and the final repair after branched TEVAR docking is shown in Figure 2A,B.

\section{Data/statistical methods}

Patients were followed prospectively with routine follow-up 

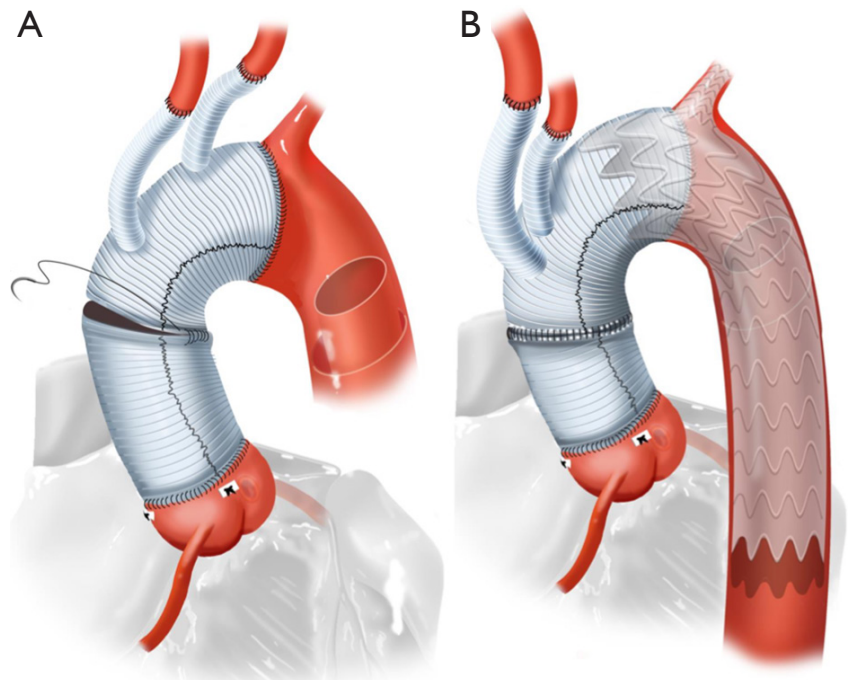

Figure 2 Open zone 2 arch for repair of acute type A dissection (A) and completed repair with branched TEVAR graft docking into aortic graft and LSCA (B) panel. TEVAR, thoracic endovascular aortic repair.

imaging. All CT scans were performed with contrast using a dissection protocol with delayed imaging and reconstructed in 3D for analysis (M2S, West Lebanon, NH). Data was managed in a prospective database and standard univariate and survival techniques were employed.

\section{Results}

Patient demographic information is presented in Table 1 . Patients were male and acute dissection patients tended to be younger. All five acute patients had complex arch dissection with large tears in the arch itself or in the descending aorta distal to the LSCA. Limb malperfusion was present in one patient, renal malperfusion in two patients, and three patients had pre-operative neurologic symptoms. Average circulatory arrest/ACP time was $49 \pm 9 \mathrm{~min}$ and the cardiopulmonary bypass time was $254 \pm 28 \mathrm{~min}$. Median days between the open repair and the zone 2 branched TEVAR were 19 days. One proximal aortic extension cuff was used to facilitate adequate overlap into the surgical graft.

There were no perioperative mortalities, strokes, or spinal cord ischemia in any patients at either stage of the procedure. There were no 30-day readmissions. Intraoperatively and at follow-up CTA, no patients had antegrade flow into the false lumen. There were no

\begin{tabular}{ll}
\hline Table 1 Patient characteristics & \\
\hline Characteristics & Zone 2 acute dissection \\
\hline $\mathrm{N}$ & 5 \\
\hline Sex (male) & $5(100 \%)$ \\
\hline Age (mean \pm SD) & $55.6 \pm 6.1$ \\
\hline Hypertension & $5(100 \%)$ \\
\hline Diabetes & $1(20 \%)$ \\
\hline Smoking Hx & $2(40 \%)$ \\
\hline Family Hx cardiac disease & $2(40 \%)$ \\
\hline COPD & $1(20 \%)$ \\
\hline Chronic renal insufficiency & $0(0 \%)$ \\
\hline Stroke history & $2(40 \%)$ \\
\hline Prior ascending dissection repair & $0(0 \%)$ \\
\hline
\end{tabular}

$\mathrm{SD}$, standard deviation; COPD, chronic obstructive pulmonary disease.

instances of new dialysis, tracheostomy, or recurrent laryngeal nerve injury. Median follow-up time for last CTA was 6 months. All patients experienced false lumen thrombosis in the stented portion of the aorta and had continued flow in the false lumen below the stented portion from distal reentry tears.

\section{Discussion}

In the current series, we describe a highly versatile approach to the management of acute DeBakey I dissection that can be used for a wide variety of dissection-related problems. The technique was originally developed out of necessity in an extremely complex acute arch dissection case in which a patient with a dissected arch and bovine trunk presented with ECG changes and chest pain (Figure $3 A, B)$. There was a small proximal tear in the RCA origin and a very large tear several centimeters distal to the LSCA orifice. The distal, primary, tear site was inaccessible from the sternotomy incision, and the decision was made to perform a zone 2 arch replacement with a two-branched graft to the innominate and left carotid arteries. This was done with a plan for completion of the arch/descending thoracic repair using the zone $2 \mathrm{graft}$ as a platform for landing the TEVAR. The patient tolerated the initial operation well but on early post-operative imaging was found to have early rapid expansion of the 

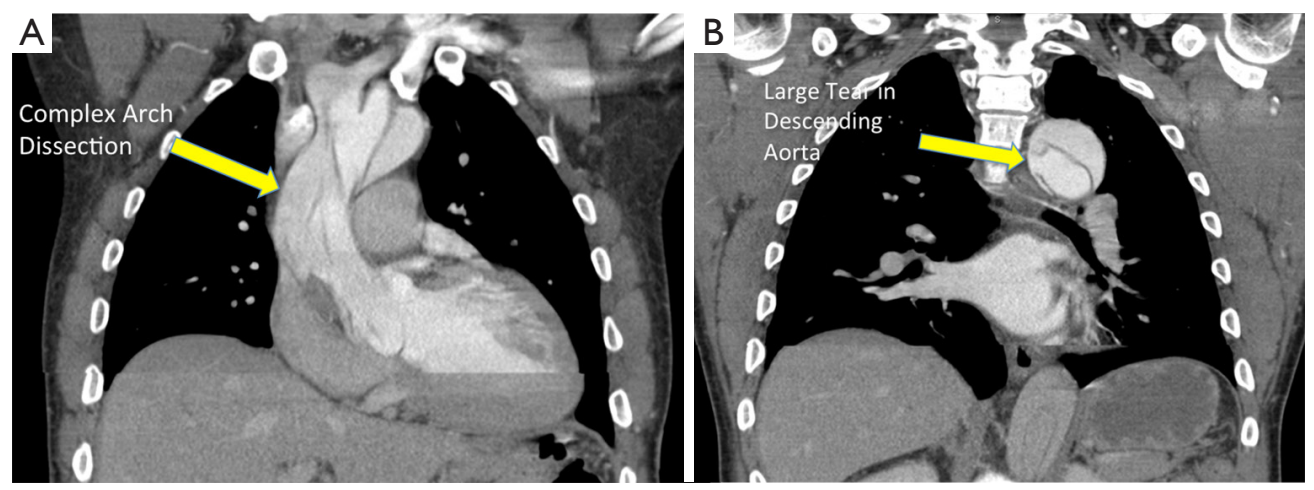

Figure 3 Acute type A dissection with complex arch flap (A) and large tear in descending thoracic aorta (B).
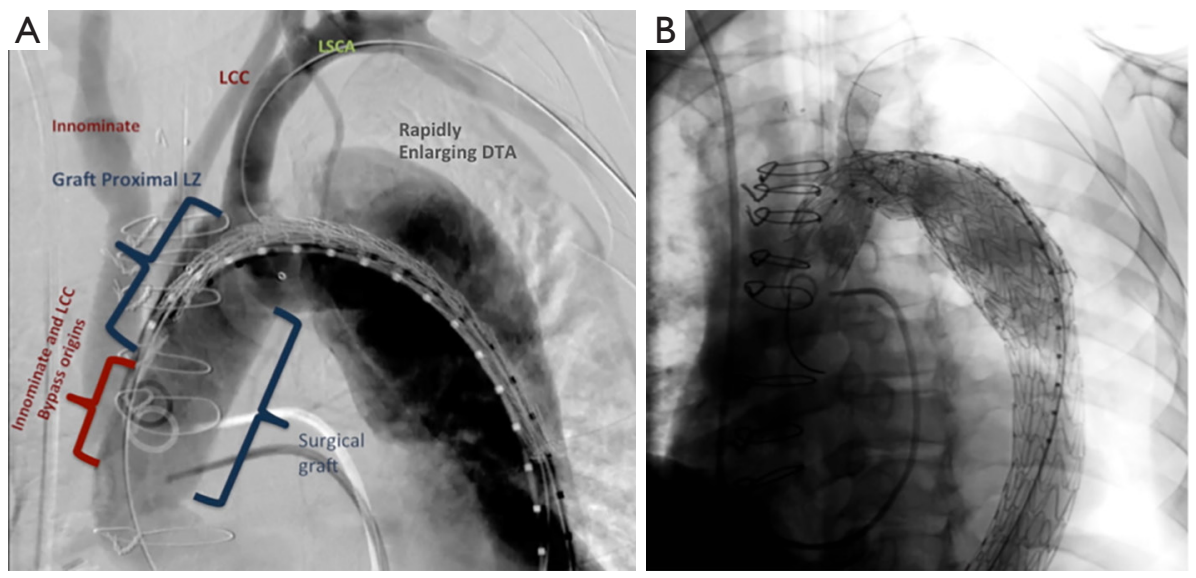

Figure 4 Angiogram of open zone 2 arch for repair of acute type A dissection (A) and completed repair with branched TEVAR graft docking into aortic graft and LSCA (B). TEVAR, thoracic endovascular aortic repair; LSCA, left subclavian artery.

proximal descending aorta and severe compression of the true lumen. This likely occurred due to the presence of the uncorrected primary tear in the descending aorta. We made the decision to perform early TEVAR to prevent potential rupture. To achieve docking of the TEVAR graft into the surgical arch graft, the LSCA origin needed to be fully covered by the TEVAR graft.

Our general approach is to always revascularize the LSCA in these cases, and we have previously done this with interval carotid subclavian bypasses. In this case, the residual dissection in the distal left carotid artery made traditional left carotid-LSCA bypass more hazardous and we wished to avoid manipulation of the dissected left carotid artery. Hence, under an emergency use protocol with the FDA, we used an investigational single branched TEVAR graft with the branch placed into the LSCA. This was then extended with standard TEVAR grafts to facilitate further remodeling into the distal aorta. Our initial experience with this procedure was extremely satisfactory, with complete exclusion of antegrade flow from the residual entry tear and remodeling of the aorta down to the distal extent of the TEVAR graft. Angiograms of the completed open repair and final repair after branched TEVAR are presented in Figure $4 A, B$.

There are several options for dealing with the arch during acute Type A dissection repair. Standard open hemi-arch reconstruction is fairly straightforward and can be performed safely by most heart surgeons. This technique has the shortest predicted circulatory arrest times and can be performed with anterograde or retrograde cerebral perfusion or even without perfusion safely (11-13). Unfortunately, this technique usually does not address arch or distal entry tears and does not allow for definitive TEVAR therapies for distal disease. Residual 
malperfusion syndromes are also possible in this situation if there is persistent arch dissection and descending aortic true lumen compression. Long-term follow-up studies have shown this approach carries a $20-40 \%$ risk of need for re-intervention in the residual arch or descending thoracic aorta (14-16). Studies examining this issue have been confounded by two issues- first, the inclusion of DeBakey II dissections, in whom the natural history of the downstream aorta is fundamentally different, and second, the substantial competing risk of death in these patients. Indeed, several series have shown 30-50\% mortality at 10 years in acute dissection patients $(17,18)$. This implies that a substantial number of patients likely died from aortic complications without reintervention and the potential risk of reinterventions among survivors, if followed more closely, is highly underestimated.

The zone 2 partial arch surgical repair presented here has several advantages for routine use in complex acute DeBakey 1 dissection. The distal aortic anastomosis between the left common carotid artery and the LSCA is relatively simple and not significantly more difficult than an aggressive hemi-arch anastomosis. This technique can address most complex arch tears, eliminate dissection flap in the proximal head vessels, and allow for later definitive TEVAR options. By avoiding an anastomosis in zone 3, there is a substantially lower risk of left recurrent laryngeal nerve injury, which may be as high as $10 \%$ in a zone 3 arch procedure (19). Deferring the TEVAR as a second procedure helps limit circulatory arrest periods associated with TEVAR deployment and limits paraplegia risk. The second stage completion with the branched graft into the left subclavian is somewhat more technically challenging than a traditional TEVAR, but was performed without any major complications. This early series shows the technical feasibility and safety of this procedure, as well as shorter circulatory arrest times compared to the hemi-arch plus antegrade TEVAR or total arch and frozen elephant trunk approaches.

\section{Conclusions}

In summary, we present an early experience of using a branched thoracic aortic endograft for DeBakey I aortic dissection in conjunction with open techniques. The procedure is highly adaptable to a range of acute dissection pathologies. In this pioneer series, complication rates were low and compared favorably with hybrid techniques developed for similar pathologies. Further study of these novel techniques is warranted.

\section{Acknowledgements}

None.

\section{Footnote}

Conflicts of Interest: Drs. Bavaria, Desai, Szeto and Wang are investigators in the Gore TBE early feasibility trial. Drs. Bavaria and Desai are consultants to WL Gore and Associates. The other authors have no conflicts of interest to declare.

\section{References}

1. Rylski B, Bavaria JE, Milewski RK, et al. Long-term results of neomedia sinus Valsalva repair in 489 patients with type A aortic dissection. Ann Thorac Surg 2014;98:582-8

2. Andersen ND, Ganapathi AM, Hanna JM, et al. Outcomes of acute type a dissection repair before and after implementation of a multidisciplinary thoracic aortic surgery program. J Am Coll Cardiol 2014;63:1796-803.

3. Crawford ES, Kirklin JW, Naftel DC, et al. Surgery for acute dissection of ascending aorta. Should the arch be included? J Thorac Cardiovasc Surg 1992;104:46-59.

4. Li B, Ma WG, Liu YM, et al. Is extended arch replacement justified for acute type A aortic dissection? Interact Cardiovasc Thorac Surg 2015;20:120-6.

5. Kim JB, Chung CH, Moon DH, et al. Total arch repair versus hemiarch repair in the management of acute DeBakey type I aortic dissection. Eur J Cardiothorac Surg 2011;40:881-7.

6. Rice RD, Sandhu HK, Leake SS, et al. Is Total Arch Replacement Associated With Worse Outcomes During Repair of Acute Type A Aortic Dissection? Ann Thorac Surg 2015;100:2159-65; discussion 2165-6.

7. Di Eusanio M, Berretta P, Cefarelli M, et al. Total Arch Replacement Versus More Conservative Management in Type A Acute Aortic Dissection. Ann Thorac Surg 2015;100:88-94.

8. Rylski B, Beyersdorf F, Desai ND, et al. Distal aortic reintervention after surgery for acute DeBakey type I or II aortic dissection: open versus endovascular repair. Eur J Cardiothorac Surg 2015;48:258-63.

9. Patel HJ, Dake MD, Bavaria JE, et al. Branched Endovascular Therapy of the Distal Aortic Arch: Preliminary Results of the Feasibility Multicenter Trial of 
the Gore Thoracic Branch Endoprosthesis. Ann Thorac Surg 2016;102:1190-8.

10. W.L. Gore and Associates. Feasibility Study for GORE® TAG® Thoracic Branch Endoprosthesis to Treat Proximal Descending Thoracic Aortic Aneurysms. In: ClinicalTrials.gov (Internet). Bethesda (MD): National Library of Medicine (US). 2000- Jun 1 2016. Available online: https://clinicaltrials.gov/ct2/show/NCT02021812

11. Preventza O, Simpson KH, Cooley DA, et al. Unilateral versus bilateral cerebral perfusion for acute type A aortic dissection. Ann Thorac Surg 2015;99:80-7.

12. Etz CD, von Aspern K, da Rocha E Silva J, et al. Impact of perfusion strategy on outcome after repair for acute type a aortic dissection. Ann Thorac Surg 2014;97:78-85.

13. Krüger T, Weigang E, Hoffmann I, et al. Cerebral protection during surgery for acute aortic dissection type A: results of the German Registry for Acute Aortic Dissection Type A (GERAADA). Circulation 2011;124:434-43.

14. Geirsson A, Bavaria JE, Swarr D, et al. Fate of the residual distal and proximal aorta after acute type a dissection repair using a contemporary surgical reconstruction

Cite this article as: Desai ND, Hoedt A, Wang G, Szeto WY, Vallabhajosyula P, Reinke M, Bavaria JE. Simplifying aortic arch surgery: open zone 2 arch with single branched thoracic endovascular aortic repair completion. Ann Cardiothorac Surg 2018;7(3):351-356. doi: 10.21037/acs.2018.05.08 algorithm. Ann Thorac Surg 2007;84:1955-64; discussion 1955-64.

15. Kirsch M, Soustelle C, Houël R, et al. Risk factor analysis for proximal and distal reoperations after surgery for acute type A aortic dissection. J Thorac Cardiovasc Surg 2002;123:318-25.

16. Zierer A, Voeller RK, Hill KE, et al. Aortic enlargement and late reoperation after repair of acute type A aortic dissection. Ann Thorac Surg 2007;84:479-86; discussion 486-7.

17. Rylski B, Milewski RK, Bavaria JE, et al. Long-term results of aggressive hemiarch replacement in 534 patients with type A aortic dissection. J Thorac Cardiovasc Surg 2014;148:2981-5.

18. Roselli EE, Loor G, He J, et al. Distal aortic interventions after repair of ascending dissection: the argument for a more aggressive approach. J Thorac Cardiovasc Surg 2015;149:S117-24.e3.

19. Neequaye $S$, Abraham CZ. Total endograft replacement of aortic arch. Ann Cardiothorac Surg 2013;2:362-6. 